Pacific Journal of Mathematics

THE 2-CLASS GROUP OF BIQUADRATIC FIELDS. II 


\section{THE 2-CLASS GROUP OF BIQUADRATIC FIELDS, II}

\section{Ezra Brown and Charles J. Parry}

We describe methods for determining the exact power of 2 dividing the class number of certain cyclic biquadratic number fields. In a recent article, we developed a relative genus theory for cyclic biquadratic fields whose quadratic subfields have odd class number; we considered the case in which the quadratic subfield is $Q(\sqrt{l})$ with $l \equiv 5(\bmod 8)$ a prime. Here we shall extend our methods to the cases in which the subfield is $Q(\sqrt{2})$ or $Q(\sqrt{l})$ with $l \equiv 1(\bmod 8)$ a prime. We consider all such cases for which the 2-class group of the biquadratic field is of rank at most 3 .

2. Notation and preliminaries.

$Q$ : the field of rational numbers.

$l$ : a rational prime satisfying $l=2$ or $l \equiv 1(\bmod 8)$.

$p, q, p_{i}$ : rational primes.

$k$ : the quadratic field $Q(\sqrt{l})$.

$\varepsilon=(u+v \sqrt{l}) / 2$, the fundamental unit of $k$, with $u, v>0$.

$m$ : a square-free positive rational integer, relatively prime to $l$. $d=-m \sqrt{l} \varepsilon$.

$K$ : the biquadratic field $k(\sqrt{d})$.

$h, h_{0}$ : the class numbers of $K$ and $k$, respectively.

$\left(\frac{x, y}{\pi}\right)$ : the quadratic norm residue symbol over $k$.

$\left[\frac{\alpha}{\beta}\right]$ : the quadratic residue symbol for $k$.

$\left(\frac{a}{b}\right)$ : the rational quadratic residue (Legendre) symbol.

$\left(\frac{a}{b}\right)_{4}$ : the rational 4 th power residue symbol (defined if and only if $(a / b)=1)$.

$N()$ : the relative norm for $K / k$.

$H$ : the 2-Sylow subgroup of the class group of $K$.

It is easy to see that $K$ is a cyclic extension of $Q$ of degree 4 which contains $k$. Recall that $\varepsilon$ has (absolute) norm -1 , that $h_{0}$ is odd and that $H$ has rank $t-1$, where $t$ is the number of prime ideals of $k$ which ramify in $K$.

3. Class number divisibility: The case $l \equiv 1(\bmod 8)$.

THEOREM 1. Let $m=p \equiv 3(\bmod 4)$. Then 


$$
\begin{aligned}
h & \equiv 2(\bmod 4) \quad \text { if } \quad\left(\frac{p}{l}\right)=-1 ; \\
& \equiv 4(\bmod 8) \quad \text { if } \quad\left(\frac{p}{l}\right)_{4}=-1 ; \\
& \equiv 0(\bmod 16) \quad \text { if } \quad\left(\frac{p}{l}\right)_{4}=1 .
\end{aligned}
$$

Proof. The number $t$ of prime ideals of $k$ which ramify in $K$ is equal to 2 or 3 according as $(p / l)=-1$ or 1 . In the first case,

$$
\left(\frac{p, d}{\sqrt{l}}\right)=\left[\frac{p}{\sqrt{l}}\right]=\left(\frac{p}{l}\right)=-1,
$$

so that only the principal ambiguous class is in the principal genus. By Theorem 1 of [1] we have $H \simeq Z_{2}$.

If $(p / l)=1$, then $p=\pi_{1} \pi_{2}$, where $\pi_{1}$ and $\pi_{2}$ are prime ideals of k. The ideals $\pi_{1}^{h_{0}}$ and $\pi_{2}^{h_{0}}$ are principal ideals, and

$$
\begin{aligned}
& \pi_{1}^{h_{0}}=a+b \sqrt{l}>0, \\
& \pi_{2}^{h_{0}}=a-b \sqrt{l}>0 .
\end{aligned}
$$

Thus,

$$
\begin{aligned}
\left(\frac{a+b \sqrt{l}, d}{\sqrt{l}}\right) & =\left[\frac{a+b \sqrt{l}}{\sqrt{l}}\right]=\left(\frac{a}{l}\right) \\
& =\left(\frac{a^{2}}{l}\right)_{4}=\left(\frac{p}{l}\right)_{4} .
\end{aligned}
$$

Also,

$$
\left(\frac{a+b \sqrt{l}, d}{\pi_{2}}\right)=\left[\frac{a+b \sqrt{l}}{\pi_{2}}\right]=\left(\frac{2 a}{p}\right) .
$$

Because $p \equiv 3(\bmod 4)$ and $h_{0}$ is odd, $a$ is even; if $a=2^{i} c$ with $c$ odd, then $i=1$ if and only if $p \equiv 3(\bmod 8)$. Thus,

$$
\begin{aligned}
\left(\frac{2 a}{p}\right) & =\left(\frac{2}{p}\right)^{i+1}\left(\frac{c}{p}\right)=\left(\frac{c}{p}\right)=\left(\frac{-p}{c}\right) \\
& =\left(\frac{l}{c}\right)=\left(\frac{c}{l}\right)=\left(\frac{c^{2}}{l}\right)_{4}=\left(\frac{a^{2}}{l}\right)_{4}=\left(\frac{p}{l}\right)_{4} .
\end{aligned}
$$

We then have the following table of characters:

\begin{tabular}{c|c|c|c}
\hline Norm\Character & $\sqrt{l}$ & $\pi_{1}$ & $\pi_{2}$ \\
\hline$\varepsilon \sqrt{l}$ & 1 & $\left(\frac{p}{l}\right)_{4}$ & $\left(\frac{p}{l}\right)_{4}$ \\
$a+b \sqrt{l}$ & $\left(\frac{p}{l}\right)_{4}$ & 1 & $\left(\frac{p}{l}\right)_{4}$ \\
$a-b \sqrt{l}$ & $\left(\frac{p}{l}\right)_{4}$ & $\left(\frac{p}{l}\right)_{4}$ & 1 \\
\hline
\end{tabular}


If $(p / l)_{4}=-1$, then only the principal ambiguous class is in the principal genus; by Theorem 1 of [1], we have $H \simeq Z_{2} \times Z_{2}$, so that $h \equiv 4(\bmod 8)$.

If $(p / l)_{4}=1$, then all four ambiguous classes are in the principal genus, so that $h \equiv 0(\bmod 16)$.

THEOREM 2. Let $m=p_{1} p_{2} \cdots p_{t} \equiv 3(\bmod 4)$ with $\left(p_{\imath} / l\right)=-1$ for all $i$. Then

$$
h \equiv 2^{t}\left(\bmod 2^{t+1}\right)
$$

Proof. $H$ has rank $t$, so we just need to show that the only ambiguous class in the principal genus is the principal class. Now

$$
\begin{aligned}
& \left(\frac{p_{i}, d}{\sqrt{l}}\right)=\left[\frac{p_{i}}{\sqrt{l}}\right]=\left(\frac{p_{i}}{l}\right)=-1, \quad \text { and } \\
& \left(\frac{p_{i}, d}{p_{j}}\right)=\left[\frac{p_{i}}{p_{j}}\right]=1 \text { for } i \neq j .
\end{aligned}
$$

It follows that $\left(p_{i}, d / p_{i}\right)=-1$ and $\left(\varepsilon \sqrt{l}, d / p_{i}\right)=-1$, by the product rule. Thus, no two of the ramified prime ideals belong to the same genus, and so the desired result follows.

THEOREM 3. Let $m=p q \equiv 3(\bmod 4)$ with $(p / l)=1$ and $(q / l)=$ -1 . Then

$$
\begin{aligned}
h & \equiv 8(\bmod 16) \quad \text { if }\left(\frac{p}{l}\right)_{4} \neq\left(\frac{q}{p}\right) ; \\
& \equiv 16(\bmod 32) \quad \text { if } p \equiv 1(\bmod 4) \quad \text { and }\left(\frac{p}{l}\right)_{4}=\left(\frac{q}{p}\right)_{1} \neq\left(\frac{l}{p}\right)_{4} ; \\
& \equiv 0(\bmod 32) \quad \text { if either } p \equiv 3(\bmod 4) \quad \text { and }\left(\frac{p}{l}\right)_{4}=\left(\frac{q}{p}\right), \\
& \text { or } p \equiv 1(\bmod 4) \text { and }\left(\frac{p}{l}\right)_{4}=\left(\frac{q}{p}\right)=\left(\frac{l}{p}\right)_{4} .
\end{aligned}
$$

Proof. Here $H$ has rank 3. Using the notation of Theorem 1, we have that

$$
\left(\frac{a+b \sqrt{l}, d}{\pi_{2}}\right)=\left[\frac{a+b \sqrt{l}}{\pi_{2}}\right]=\left[\frac{2 a}{\pi_{2}}\right]=\left(\frac{2 a}{p}\right) .
$$

If $p \equiv 3(\bmod 4)$, then $(2 a / p)=(p / l)_{4}$, as before. However, if $p \equiv 1$ $(\bmod 4)$, then

$$
\left(\frac{2 a}{p}\right)=\left(\frac{2}{p}\right)\left(\frac{a}{p}\right)=\left(\frac{2}{p}\right)\left(\frac{a^{2}}{p}\right)_{4}=\left(\frac{2}{p}\right)\left(\frac{b}{p}\right)\left(\frac{l}{p}\right)_{4} .
$$


Now $b=2^{i} c$ with $c$ odd; furthermore, $i=1$ if and only if $p \equiv 5$ $(\bmod 8)$. Hence,

$$
\left(\frac{2}{p}\right)\left(\frac{b}{p}\right)=\left(\frac{2}{p}\right)^{i+1}\left(\frac{c}{p}\right)=\left(\frac{c}{p}\right)=\left(\frac{p}{c}\right)=\left(\frac{a^{2}}{c}\right)=1 ;
$$

we deduce that $(2 a / p)=(l / p)_{4}$. Furthermore,

$$
\begin{aligned}
& \left(\frac{a+b \sqrt{l}, d}{q}\right)=\left[\frac{a+b \sqrt{l}}{q}\right]=\left(\frac{p}{q}\right)=\left(\frac{q}{p}\right), \text { and } \\
& \left(\frac{q, d}{\pi_{1}}\right)=\left[\frac{q}{\pi_{1}}\right]=\left(\frac{q}{p}\right) \text {. }
\end{aligned}
$$

The remaining characters are easily evaluated; if we set $(l / p)_{4}=(p / l)_{4}$ if $p \equiv 3(\bmod 4)$, we have the following table of characters:

\begin{tabular}{c|c|c|c|c}
\hline Norm|Character & $\sqrt{l}$ & $q$ & $\pi_{1}$ & $\pi_{2}$ \\
\hline$\varepsilon \sqrt{l}$ & -1 & -1 & $\left(\frac{p}{l}\right)_{4}$ & $\left(\frac{p}{l}\right)_{4}$ \\
$q$ & -1 & -1 & $\left(\frac{q}{p}\right)$ & $\left(\frac{q}{p}\right)$ \\
$a+b \sqrt{l}$ & $\left(\frac{p}{l}\right)_{4}$ & $\left(\frac{q}{p}\right)$ & $\left(\frac{q}{p}\right)\left(\frac{p}{l}\right)_{4}\left(\frac{l}{p}\right)_{4}$ & $\left(\frac{l}{p}\right)_{4}$ \\
$a-b \sqrt{l}$ & $\left(\frac{p}{l}\right)_{4}$ & $\left(\frac{q}{p}\right)$ & $\left(\frac{l}{p}\right)_{4}$ & $\left(\frac{q}{p}\right)\left(\frac{p}{l}\right)_{4}\left(\frac{l}{p}\right)_{4}$ \\
\hline
\end{tabular}

The theorem follows, as before, from an analysis of the various cases.

THEOREM 4. Let $m=p \equiv 1(\bmod 4)$ with $(p / l)=-1$. Then

$$
\begin{aligned}
h & \equiv 8(\bmod 16) \quad \text { if }\left(\frac{2}{l}\right)_{4} \neq\left(\frac{2}{p}\right) ; \\
& \equiv 16(\bmod 32) \quad \text { if }\left(\frac{2}{l}\right)_{4}=\left(\frac{2}{p}\right)=(-1)^{(l+7) / 8} ; \\
& \equiv 0(\bmod 32) \quad \text { if } \quad\left(\frac{2}{l}\right)_{4}=\left(\frac{2}{p}\right)=(-1)^{(l-1) / 8} .
\end{aligned}
$$

Proof. Here, the two prime divisors of 2 in $k$ ramify in $K$. Put $2=2_{1} 2_{2}$ in $k$, with

$$
2_{1}^{h_{0}}=\alpha=\frac{a+b \sqrt{l}}{2}>0,
$$

and 


$$
2_{1}^{h_{0}}=\bar{\alpha}=\frac{a-b \sqrt{l}}{2}>0
$$

Then

$$
\begin{aligned}
\left(\frac{\alpha, d}{\sqrt{l}}\right) & =\left[\frac{\alpha}{\sqrt{l}}\right]=\left[\frac{a / 2}{\sqrt{l}}\right]=\left(\frac{2 a}{l}\right) \\
& =\left(\frac{4 a^{2}}{l}\right)_{4}=\left(\frac{2}{l}\right)_{4}, \\
\left(\frac{\alpha, d}{p}\right) & =\left[\frac{\alpha}{p}\right]=\left(\frac{2}{p}\right), \text { and } \\
\left(\frac{p, d}{2_{1}}\right) & =(-1)^{(p-1) / 2}=1 \text {. Now } \\
{\left[\frac{a+b \sqrt{l}}{2}\right]^{2} } & =\frac{1}{2}\left(a^{2}-2^{h_{0}+1}+a b \sqrt{l}\right), \text { so that } \\
a \bar{\alpha} & \equiv \frac{1}{2}\left(a^{2}-a b \sqrt{l}\right) \equiv a^{2}-2^{h_{0}}\left(\bmod 2_{1}^{2}\right) \text {. Thus, } \\
\left(\frac{\bar{\alpha}, d}{2_{1}}\right) & =\left(\frac{a, d}{2_{1}}\right)\left(\frac{a^{2}-2^{h_{0}}, d}{2_{1}}\right) \\
& =(-1)^{(a-1) / 2}(-1)^{\left(a^{2}-2^{\left.h_{0}-1\right) / 2}\right.} \\
& =\left(\frac{-1}{a}\right)(-1)^{2^{h_{0}-1}} \cdot
\end{aligned}
$$

To evaluate $(-1 / a)$, note that

$$
\left(\frac{a}{l}\right)=\left(\frac{a^{2}}{l}\right)_{4}=\left(\frac{2}{l}\right)_{4}
$$

and

$$
\left(\frac{2}{a}\right)=\left(\frac{-l}{a}\right)=\left(\frac{-1}{a}\right)\left(\frac{l}{a}\right)=\left(\frac{-1}{a}\right)\left(\frac{a}{l}\right) .
$$

Hence,

$$
\left(\frac{-1}{a}\right)=\left(\frac{2}{a}\right)\left(\frac{a}{l}\right)=\left(\frac{2}{a}\right)\left(\frac{2}{l}\right)_{4} .
$$

Since $(2 / b)=1$, we have $b^{2} \equiv 1(\bmod 16)$, so that

$$
a^{2}-l b^{2} \equiv a^{2}-l \equiv 2^{h_{0}+2}(\bmod 16) .
$$

If $h_{0}=1$, then $a^{2} \equiv l+8(\bmod 16)$, so that

$$
\left(\frac{2}{a}\right)=1 \text { if and only if } l \equiv 9(\bmod 16) ;
$$


if $h_{0}>1$, then $a^{2} \equiv l(\bmod 16)$, so that

$$
\left(\frac{2}{a}\right)=1 \quad \text { if and only if } l \equiv 1(\bmod 16) .
$$

In either case,

$$
\left(\frac{\bar{\alpha}, d}{2_{1}}\right)=(-1)^{2^{h_{0-1}}}\left(\frac{-1}{a}\right)=(-1)^{(l-1) / 8}\left(\frac{2}{l}\right)_{4} .
$$

Finally, we note that

$$
\left(\frac{p, d}{\sqrt{l}}\right)=\left(\frac{p, d}{p}\right)=-1 .
$$

This yields the following table of generic characters:

\begin{tabular}{c|c|c|c|c}
\hline Norm|Characters & $\sqrt{l}$ & $p$ & $2_{1}$ & $2_{2}$ \\
\hline$p$ & -1 & -1 & +1 & +1 \\
$\alpha$ & $\left(\frac{2}{l}\right)_{4}$ & $\left(\frac{2}{p}\right)$ & $(-1)^{(l-1) / 8}\left(\frac{2}{p}\right)$ & $(-1)^{(l-1) / 8}\left(\frac{2}{l}\right)_{4}$ \\
$\bar{\alpha}$ & $\left(\frac{2}{l}\right)_{4}$ & $\left(\frac{2}{p}\right)$ & $(-1)^{(l-1) / 8}\left(\frac{2}{l}\right)_{4}$ & $(-1)^{(l-1) / 8}\left(\frac{2}{p}\right)$ \\
\hline
\end{tabular}

If $(2 / l)_{4} \neq(2 / p)$, then all three lines of the table are distinct and only the principal ambiguous class lies in the principal genus; this implies that $h \equiv 8(\bmod 16)$.

If $(2 / l)_{4}=(2 / p) \neq(-1)^{(l-1) / 8}$, then the last two lines are identical, but different from the first. Here, exactly two ambiguous classes lie in the principal genus, and so $h \equiv 16(\bmod 32)$.

In the case $(2 / l)_{4}=(2 / p)=(-1)^{(l-1) / 8}$, there are 4 ambiguous classes in the principal genus. Thus $h \equiv 0(\bmod 32)$.

Corollary. If $m=1$, then

$$
\begin{aligned}
h & \equiv 4(\bmod 8) \quad \text { if } \quad\left(\frac{2}{l}\right)_{4}=-1 ; \\
& \equiv 8(\bmod 16) \quad \text { if } \quad l \equiv 9(\bmod 16) \text { and } \quad\left(\frac{2}{l}\right)_{4}=1 ; \\
& \equiv 0(\bmod 16) \quad \text { if } \quad l \equiv 1(\bmod 16) \text { and } \quad\left(\frac{2}{l}\right)_{4}=1 .
\end{aligned}
$$

Proof. Here $t=3$ and so $H$ has rank 2. The table of generic characters is obtained by setting $(2 / p)=1$ in the last two lines of 
the table in Theorem 4. There are 1,2 or 4 ambiguous classes in the principal genus according as the condition of the first, second or third line of the corollary holds.

THEOREM 5. If $m=2$, then

$$
\begin{aligned}
h & \equiv 4(\bmod 8), \quad \text { if }\left(\frac{2}{l}\right)_{4}=-1 ; \\
& \equiv 0(\bmod 16), \quad \text { if }\left(\frac{2}{l}\right)_{4}=1 .
\end{aligned}
$$

Proof. Using the notation of the preceding theorem, we have

$$
\begin{aligned}
\left(\frac{\bar{\alpha}, d}{2_{1}}\right) & =\left(\frac{\bar{\alpha},-2 \varepsilon \sqrt{l}}{2_{1}}\right)=\left(\frac{\bar{\alpha}, 2}{2_{1}}\right)\left(\frac{\bar{\alpha},-\varepsilon \sqrt{l}}{2_{1}}\right) \\
& =\left(\frac{\bar{\alpha}, 2}{2_{1}}\right)(-1)^{(l-1) / 8}\left(\frac{2}{l}\right)_{4},
\end{aligned}
$$

the last step following from the calculations of Theorem 4. Now

$$
\alpha^{3}=\left(\frac{a+b \sqrt{l}}{2}\right)^{3}=\left(\frac{1}{2}\right)\left(a\left(a^{2}-3 \cdot 2^{h_{0}}\right)+b\left(a^{2}-2^{h_{0}}\right) \sqrt{l}\right),
$$

so that

$$
\begin{aligned}
\left(\frac{\bar{\alpha}, 2}{2_{1}}\right) & =\left(\frac{a^{2}-2^{h_{0}}, 2}{2_{1}}\right)\left(\frac{a\left(a^{2}-2^{h_{0}+1}\right), 2}{2_{1}}\right) \\
& =\left(\frac{2}{a^{2}-2^{h_{0}}}\right)\left(\frac{2}{a}\right)\left(\frac{2}{a^{2}-2^{h_{0}+1}}\right) \\
& =(-1)^{2^{h_{0}-1}}\left(\frac{2}{a}\right)=(-1)^{\{l-1\rangle / 8}
\end{aligned}
$$

Hence,

$$
\left(\frac{\bar{\alpha}, d}{2_{1}}\right)=(-1)^{(l-1) / 8}(-1)^{(l-1) / 8}\left(\frac{2}{l}\right)_{4}=\left(\frac{2}{l}\right)_{4} .
$$

We obtain the following table of characters and the result follows by considerations similar to those previously mentioned:

\begin{tabular}{c|c|c|c}
\hline Norm|Character & $\sqrt{l}$ & $2_{1}$ & $2_{2}$ \\
\hline$\varepsilon \sqrt{l}$ & 1 & $\left(\frac{2}{l}\right)_{4}$ & $\left(\frac{2}{l}\right)_{4}$ \\
$\alpha$ & $\left(\frac{2}{l}\right)_{4}$ & 1 & $\left(\frac{2}{l}\right)_{4}$ \\
$\bar{\alpha}$ & $\left(\frac{2}{l}\right)_{4}$ & $\left(\frac{2}{l}\right)_{4}$ & 1 \\
\hline
\end{tabular}


THEOREM 6. If $m=2 p$ with $(p / l)=-1$, then

$$
\begin{aligned}
h & \equiv 8(\bmod 16) \text { if }\left(\frac{2}{l}\right)_{4} \neq\left(\frac{2}{p}\right) ; \\
& \equiv 16(\bmod 32) \quad \text { if }\left(\frac{2}{l}\right)_{4}=\left(\frac{2}{p}\right) \neq(-1)^{(l-1) / 8}, \\
& \equiv 0(\bmod 32), \quad \text { and } p \equiv 3(\bmod 8) ;
\end{aligned}
$$

Proof. First we note that

$$
\begin{aligned}
\left(\frac{\bar{\alpha}, d}{2_{1}}\right) & =\left(\frac{\bar{\alpha},-2 p \varepsilon \sqrt{l}}{2_{1}}\right)=\left(\frac{\bar{\alpha}, 2}{2_{1}}\right)\left(\frac{\bar{\alpha},-\varepsilon p \sqrt{l}}{2_{1}}\right) \\
& =(-1)^{(l-1) / 8}\left(\frac{\bar{\alpha},-\varepsilon p \sqrt{l}}{2_{1}}\right) .
\end{aligned}
$$

If $p \equiv 1(\bmod 4)$, then the last symbol was evaluated in the proof of Theorem 4 and reduces to $(-1)^{(l-1) / 8}(2 / l)_{4}$.

If $p \equiv 3(\bmod 4)$, then $2_{1}$ is unramified in the extension $Q\left(\sqrt{d_{1}}\right)$, where $d_{1}=-\varepsilon p \sqrt{l}$. Thus, the last symbol is equal to 1 . Hence

$$
\left(\frac{\bar{\alpha}, d}{2_{1}}\right)=\left(\frac{\alpha, d}{2_{2}}\right)=\left(\frac{2}{l}\right)_{4} \text { or }(-1)^{(l-1) / 8}
$$

according as $p \equiv 1$ or $3(\bmod 4)$. Evaluation of the remaining symbols is routine, and we have the following table for $p \equiv 3(\bmod 4)$ :

\begin{tabular}{c|c|c|c|c}
\hline Norm|Character & $\sqrt{l}$ & $p$ & $2_{1}$ & $2_{2}$ \\
\hline$\varepsilon \sqrt{l}$ & -1 & -1 & $\left(\frac{2}{l}\right)_{4}$ & $\left(\frac{2}{l}\right)_{4}$ \\
$p$ & -1 & -1 & $\left(\frac{2}{p}\right)$ & $\left(\frac{2}{p}\right)$ \\
$\alpha$ & $\left(\frac{2}{l}\right)_{4}$ & $\left(\frac{2}{p}\right)$ & $(-1)^{(l-1) / 8}\left(\frac{2}{p}\right)\left(\frac{2}{l}\right)_{4}$ & $(-1)^{(l-1) / 8}$ \\
$\bar{\alpha}$ & $\left(\frac{2}{l}\right)_{4}$ & $\left(\frac{2}{p}\right)$ & $(-1)^{(l-1) / 8}$ & $(-1)^{(l-1) / 8}\left(\frac{2}{p}\right)\left(\frac{2}{l}\right)_{4}$ \\
\hline
\end{tabular}

If $p \equiv 1(\bmod 4)$, the four entries in the lower right-hand corner are replaced by

$$
\begin{array}{ll}
\left(\frac{2}{p}\right) & \left(\frac{2}{l}\right)_{4} \\
\left(\frac{2}{l}\right)_{4} & \left(\frac{2}{p}\right)^{2}
\end{array}
$$


and the desired results follow as before.

4. Class numbers divisibility: The case $l=2$.

THEOREM 7. If $m=p$, then

$$
\begin{aligned}
h & \equiv 2(\bmod 4), \text { if } p \equiv \pm 3(\bmod 8) ; \\
& \equiv 4(\bmod 8), \text { if } p \equiv \pm 7(\bmod 16) ; \\
& \equiv 8(\bmod 16), \text { if } p \equiv 1(\bmod 16) \text { and }\left(\frac{2}{p}\right)_{4}=-1 ; \\
& \equiv 0(\bmod 16), \text { if } p \equiv 1(\bmod 16) \text { and }\left(\frac{2}{p}\right)_{4}=1 \text {, or } \\
& \text { if } p \equiv 15(\bmod 16) .
\end{aligned}
$$

Proof. If $p \equiv \pm 3(\bmod 8)$ then $H$ is cyclic and

$$
\left(\frac{p, d}{\sqrt{2}}\right)=\left(\frac{2}{p}\right)=-1 \text {. }
$$

Hence, the only ambiguous class in the principal genus is the principal class, and so $H \simeq Z_{2}$.

If $p \equiv \pm 1(\bmod 8)$ then $H$ has rank 2. Let $p=\pi_{1} \pi_{2}=(a+b \sqrt{2})$ $(a-b \sqrt{2})$ with $\pi_{1}=a+b \sqrt{2}>0$. If $p \equiv 7(\bmod 8)$, then

$$
\begin{aligned}
\left(\frac{\pi_{1}, d}{\pi_{2}}\right)=\left[\frac{\pi_{1}}{\pi_{2}}\right]=\left[\frac{2 a}{\pi_{2}}\right]=\left(\frac{2 a}{p}\right)=\left(\frac{a}{p}\right) \\
=\left(\frac{-1}{a}\right)\left(\frac{p}{a}\right)=\left(\frac{-1}{a}\right)\left(\frac{-2 b^{2}}{a}\right) \\
=\left(\frac{2}{a}\right)=(-1)^{\left(a^{2}-1\right) / 8}=(-1)^{\left(p+2 b^{2}-1\right) / 8} \\
=(-1)^{(p+1) / 8},
\end{aligned}
$$

since $b$ must be odd. Furthermore,

$$
b \varepsilon \sqrt{2}=2 b+b \sqrt{2} \equiv 2 b-a\left(\bmod \pi_{1}\right),
$$

so that

$$
b^{2} \varepsilon \sqrt{2} \equiv 2 b^{2}-a b \equiv a^{2}-a b \equiv a(a-b)\left(\bmod \pi_{1}\right) .
$$

Thus,

$$
\left(\frac{\varepsilon \sqrt{2}, d}{\pi_{1}}\right)=\left[\frac{\varepsilon \sqrt{2}}{\pi_{1}}\right]=\left(\frac{a(a-b)}{p}\right)=\left(\frac{a}{p}\right)\left(\frac{a-b}{p}\right) .
$$

But $(a-b)(a+b)=a^{2}-b^{2}=p+b^{2}$, so if $a-b=2^{i} c$ with $c$ odd, we have 


$$
\left(\frac{a-b}{p}\right)=\left(\frac{2}{p}\right)^{i}\left(\frac{c}{p}\right)=\left(\frac{c}{p}\right)=\left(\frac{-p}{c}\right)=\left(\frac{b^{2}}{c}\right)=1 \text {. }
$$

Hence,

$$
\left(\frac{\varepsilon \sqrt{2}, d}{\pi_{1}}\right)=\left(\frac{a}{p}\right)=(-1)^{(p+1) / 8} .
$$

Thus, for $p \equiv 7(\bmod 8)$, we have the following table of generic characters:

\begin{tabular}{c|c|c|c}
\hline Norm|Character & $\sqrt{\mathbf{2}}$ & $\pi_{1}$ & $\pi_{2}$ \\
\hline$\varepsilon \sqrt{2}$ & 1 & $(-1)^{(p+1) / 8}$ & $(-1)^{(p+1) / 8}$ \\
$\pi_{1}$ & $(-1)^{(p+1) / 8}$ & 1 & $(-1)^{(p+1) / 8}$ \\
$\pi_{2}$ & $(-1)^{(p+1) / 8}$ & $(-1)^{(p+1) / 8}$ & 1 \\
\hline
\end{tabular}

If $p \equiv 7(\bmod 16)$, then none of the above lines are the same, so that $h \equiv 4(\bmod 8)$; if $p \equiv 15(\bmod 16)$, then all of the above lines are the same, so that $h \equiv 0(\bmod 16)$.

Now let $p \equiv 1(\bmod 8)$. Then

$$
\begin{aligned}
\left(\frac{\pi_{1}, d}{\pi_{2}}\right) & =\left(\frac{a}{p}\right)=\left(\frac{a^{2}}{p}\right)_{4}=\left(\frac{2 b^{2}}{p}\right)_{4} \\
& =\left(\frac{2}{p}\right)_{4}\left(\frac{b}{p}\right) .
\end{aligned}
$$

Setting $b=2^{i} c$ with $c$ odd, we have

$$
\left(\frac{b}{p}\right)=\left(\frac{2}{p}\right)^{i}\left(\frac{c}{p}\right)=\left(\frac{c}{p}\right)=\left(\frac{p}{c}\right)=\left(\frac{a^{2}}{c}\right)=1 .
$$

Hence,

$$
\left(\frac{\pi_{1}, d}{\pi_{2}}\right)=\left(\frac{\pi_{2}, d}{\pi_{1}}\right)=\left(\frac{2}{p}\right)_{4}
$$

Now

$$
\left(\frac{\varepsilon \sqrt{2}, d}{\pi_{2}}\right)=\left(\frac{a}{p}\right)\left(\frac{a-b}{p}\right)=\left(\frac{2}{p}\right)_{4}\left(\frac{a-b}{p}\right) .
$$

Since $(a-b)(a+b)=p+b^{2}$, we have

$$
\left(\frac{a-b}{p}\right)=\left(\frac{p}{a-b}\right)=\left(\frac{-b^{2}}{a-b}\right)=\left(\frac{-1}{a-b}\right) .
$$

A paper of G. Pall [2] contains a table, part of which we re- 
produce here:

$$
p=a^{2}-2 b^{2}=u^{2}+v^{2}, \quad v \text { even }
$$

$\begin{array}{cccc}p(\bmod 16) & v(\bmod 8) & a(\bmod 8) & b(\bmod 4) \\ 1 & 4 & 7 & 0 \\ 1 & 4 & 5 & 2 \\ 1 & 0 & 3 & 2 \\ 1 & 0 & 1 & 0 \\ 9 & 0 & 1 & 2 \\ 9 & 0 & 3 & 0 \\ 9 & 4 & 5 & 0 \\ 9 & 4 & 7 & 2\end{array}$

Thus, if $p \equiv 1(\bmod 16)$, then $(-1 /(a-b))=1$ if and only if $v \equiv 0(\bmod 8)$, and if $p \equiv 9(\bmod 16)$, then $(-1 /(a-b))=1$ if and only if $v \equiv 4(\bmod 8)$, so

$$
\left(\frac{-1}{a-b}\right)=(-1)^{v / 4}(-1)^{(p-1) / 8} .
$$

Now, Dirichlet's necessary and sufficient condition that $(2 / p)_{4}=1$ is that $v \equiv 0(\bmod 8)$. Hence, $(2 / p)_{4}=(-1)^{v / 4}$;

$$
\begin{aligned}
\left(\frac{\varepsilon \sqrt{ } 2, d}{\pi_{1}}\right) & =\left(\frac{a}{p}\right)\left(\frac{a-b}{p}\right)=\left(\frac{2}{p}\right)_{4}\left(\frac{-1}{a-b}\right) \\
& =\left(\frac{2}{p}\right)_{4}(-1)^{v / 4}(-1)^{(p-1) / 8} \\
& =\left(\frac{2}{p}\right)_{4}\left(\frac{2}{p}\right)_{4}(-1)^{(p-1) / 8}=(-1)^{(p-1) / 8} .
\end{aligned}
$$

We thus have the following table:

\begin{tabular}{c|c|c|c}
\hline Norm|Character & $\sqrt{2}$ & $\pi_{1}$ & $\pi_{2}$ \\
\hline$\varepsilon \sqrt{2}$ & 1 & $(-1)^{(p-1) / 8}$ & $(-1)^{(p-1) / 8}$ \\
$\pi_{1}$ & $(-1)^{(p-1) / 8}$ & $(-1)^{(p-1) / 8}\left(\frac{2}{p}\right)_{4}$ & $\left(\frac{2}{p}\right)_{4}$ \\
$\pi_{2}$ & $(-1)^{(p-1) / 8}$ & $\left(\frac{2}{p}\right)_{4}$ & $(-1)^{(p-1) / 8}\left(\frac{2}{p}\right)_{4}$ \\
\hline
\end{tabular}

If $p \equiv 9(\bmod 16)$, then each line is different; thus, only the principal ambiguous class belongs to the principal genus, and so $H \simeq Z_{2} \times Z_{2}, h \equiv 4(\bmod 8)$. 
If $p \equiv 1(\bmod 16)$, then there are either two or four ambiguous classes in the principal genus, according as $(2 / p)_{4}=-1$ or 1 . In these cases, $h \equiv 8$ or $0(\bmod 16)$, respectively.

THEOREM 8. If $m=p_{1} \cdots p_{t}$ with $\left(2 / p_{i}\right)=-1$ for all $i$, then

$$
h \equiv 2^{t}\left(\bmod 2^{t+1}\right) \text {. }
$$

Comment. The proof is quite similar to the proof of Theorem 2 , so we omit it.

THEOREM 9. Let $m=p q$ with $(2 / p)=1$ and $(2 / q)=-1$.

If $p \equiv 1(\bmod 8)$, then

$$
\begin{aligned}
h & \equiv 8(\bmod 16), \quad \text { if }\left(\frac{p}{q}\right) \neq(-1)^{(p-1) / 8} ; \\
& \equiv 16(\bmod 32), \quad \text { if }\left(\frac{2}{p}\right)_{4} \neq(-1)^{(p-1) / 8}=\left(\frac{p}{q}\right) ; \\
& \equiv 0(\bmod 32), \quad \text { otherwise. }
\end{aligned}
$$

If $p \equiv 7(\bmod 8)$, then

$$
\begin{aligned}
h & \equiv 8(\bmod 16), \quad \text { if }\left(\frac{p}{q}\right) \neq(-1)^{(p+1) / 8} ; \\
& \equiv 16(\bmod 32), \quad \text { if } q \equiv 3(\bmod 4) \text { and }\left(\frac{p}{q}\right)=(-1)^{(p+1) / 8}=-1 ; \\
& \equiv 0(\bmod 32), \quad \text { otherwise. }
\end{aligned}
$$

Comment. The proof involves straightforward extensions of the tables, constructed in the proof of Theorem 7, so we will omit it.

5. Numerical results, A slight modification of the methods described in [3] allow us to compute the relative class number $h^{*}=h / h_{0}$ of $K$. As $h_{0}=1$ for most small values of $l$, we have $h^{*}=h$ for almost all values within the range of our computations. In the tables below we list all fields within the range of our calculations, where the maximum power of dividing $h^{*}$ exceeds the power predicted in $\S 3$. We have only computed values of $h^{*}$ for the fields discussed in Theorems $1,4,5,6$, and 7 . The column of the table headed by $f$ gives the prime factorization of $h^{*}$. 


\begin{tabular}{|c|c|c|c|c|c|c|c|}
\hline \multicolumn{4}{|c|}{ Table 1} & \multicolumn{4}{|c|}{ Table 1 (con't) } \\
\hline \multicolumn{4}{|c|}{$(d=-\varepsilon \sqrt{l} p, p \equiv 3 \bmod 4)$} & \multicolumn{4}{|c|}{$(d=-\varepsilon \sqrt{l} p, p \equiv 3 \bmod 4)$} \\
\hline$l$ & $p$ & $h^{*}$ & $f$ & $l$ & $p$ & $h^{*}$ & $f$ \\
\hline \multirow[t]{4}{*}{17} & 67 & 160 & $2^{5} \cdot 5$ & 73 & 71 & 640 & $2^{7} \cdot 5$ \\
\hline & 103 & 32 & $2^{5}$ & 89 & 67 & 128 & $2^{7}$ \\
\hline & 251 & 1088 & $2^{8} \cdot 17$ & 97 & 47 & 64 & $2^{6}$ \\
\hline & 463 & 160 & $2^{5} \cdot 5$ & & 103 & 544 & $2^{5} \cdot 17$ \\
\hline \multirow[t]{10}{*}{41} & 23 & 32 & $2^{5}$ & 113 & 7 & 160 & $2^{5} \cdot 5$ \\
\hline & 59 & 288 & $2^{5} \cdot 9$ & 193 & 3 & 160 & $2^{5} \cdot 5$ \\
\hline & 83 & 1184 & $2^{5} \cdot 37$ & & 47 & 576 & $2^{6} \cdot 3^{2}$ \\
\hline & 139 & 832 & $2^{6} \cdot 13$ & 233 & 71 & 5696 & $2^{6} \cdot 89$ \\
\hline & 163 & 1312 & $2^{5} \cdot 41$ & & 107 & 800 & $2^{5} \cdot 5^{2}$ \\
\hline & 223 & 256 & $2^{8}$ & $257^{*}$ & 11 & 64 & $2^{6}$ \\
\hline & 271 & 160 & $2^{5} \cdot 5$ & & 23 & 640 & $2^{6} \cdot 5$ \\
\hline & 283 & 3328 & $2^{8} \cdot 13$ & & 67 & 416 & $2^{5} \cdot 13$ \\
\hline & 379 & 2080 & $2^{5} \cdot 5 \cdot 13$ & 281 & 59 & 160 & $2^{5} \cdot 5$ \\
\hline & 491 & 2592 & $2^{5} \cdot 3^{4}$ & & & & \\
\hline
\end{tabular}

$\left(^{*}\right) \quad h_{0}=3$ when $l=257$.

\begin{tabular}{c|c|c|c|c|c|c|c}
\hline \multicolumn{2}{c|}{ Table 2 } & \multicolumn{4}{|c|}{ Table 2 (con't) } \\
\hline \multicolumn{2}{c|}{$(d=-\varepsilon \sqrt{l} p, p \equiv 1 \bmod 4)$} & \multicolumn{2}{|c}{$(d=-\varepsilon \sqrt{l} p, p \equiv 1$ mod 4$)$} \\
\hline \multirow{2}{*}{17} & $p$ & $h^{*}$ & $f$ & $l$ & $p$ & $h^{*}$ & $f$ \\
& 149 & 320 & $2^{6} \cdot 5$ & 41 & 173 & 1856 & $2^{6} \cdot 29$ \\
& 157 & 512 & $2^{9}$ & & 181 & 1088 & $2^{6} \cdot 17$ \\
& 229 & 640 & $2^{7} \cdot 5$ & & 197 & 2048 & $2^{11}$ \\
& 293 & 640 & $2^{7} \cdot 5$ & & 229 & 1600 & $2^{6} \cdot 5^{2}$ \\
& 353 & 1024 & $2^{10}$ & & 269 & 1600 & $2^{6} \cdot 5^{2}$ \\
& 389 & 1600 & $2^{6} \cdot 5^{2}$ & & 293 & 3200 & $2^{7} \cdot 5^{2}$ \\
& 409 & 832 & $2^{6} \cdot 13$ & & 373 & 4096 & $2^{12}$ \\
& 53 & 832 & $2^{6} \cdot 13$ & & 389 & 2176 & $2^{7} \cdot 17$ \\
& 61 & 320 & $2^{6} \cdot 5$ & & 433 & 5248 & $2^{7} \cdot 41$ \\
& 109 & 576 & $2^{6} \cdot 3^{2}$ & 73 & 41 & 320 & $2^{6} \cdot 5$ \\
\hline
\end{tabular}




\begin{tabular}{|c|c|c|c|c|c|c|c|}
\hline \multicolumn{4}{|c|}{ Table 2 (con't) } & \multicolumn{4}{|c|}{ Table 2 (con't) } \\
\hline \multicolumn{4}{|c|}{$(d=-\varepsilon \sqrt{l} p, p \equiv 1 \bmod 4)$} & \multicolumn{4}{|c|}{$(d=-\varepsilon \sqrt{l} p, p \equiv 1 \bmod 4)$} \\
\hline$l$ & $p$ & $h^{*}$ & $f$ & $l$ & $p$ & $h^{*}$ & $f$ \\
\hline \multirow[t]{2}{*}{78} & 89 & 512 & $2^{9}$ & 137 & 73 & 1280 & $2^{8} \cdot 5$ \\
\hline & 109 & 2368 & $2^{6} \cdot 37$ & & 109 & 3136 & $2^{6} \cdot 7^{2}$ \\
\hline \multirow[t]{2}{*}{89} & 73 & 2560 & $2^{9} \cdot 5$ & 193 & 101 & 10816 & $2^{6} \cdot 13^{2}$ \\
\hline & 97 & 2560 & $2^{9} \cdot 5$ & 233 & 29 & 1280 & $2^{8} \cdot 5$ \\
\hline \multirow[t]{3}{*}{97} & 53 & 512 & $2^{9}$ & & 37 & 2304 & $2^{8} \cdot 3^{2}$ \\
\hline & 101 & 832 & $2^{6} \cdot 13$ & 241 & 5 & 128 & $2^{7}$ \\
\hline & 109 & 3904 & $2^{6} \cdot 61$ & & 61 & 4608 & $2^{9} \cdot 3^{?}$ \\
\hline \multirow[t]{7}{*}{113} & 17 & 320 & $2^{6} \cdot 5$ & & 97 & 16000 & $2^{7} \cdot 5^{3}$ \\
\hline & 41 & 1088 & $2^{6} \cdot 17$ & 257 & 17 & 832 & $2^{6} \cdot 13$ \\
\hline & 53 & 832 & $2^{6} \cdot 13$ & & 41 & 2560 & $2^{9} \cdot 5$ \\
\hline & 73 & 1600 & $2^{6} \cdot 5^{2}$ & & 73 & 3200 & $2^{7} \cdot 5^{2}$ \\
\hline & 89 & 3712 & $2^{7} \cdot 29$ & & 89 & 4672 & $2^{6} \cdot 73$ \\
\hline & 97 & 4352 & $2^{8} \cdot 17$ & 281 & 29 & 1600 & $2^{6} \cdot 5^{2}$ \\
\hline & 109 & 1664 & $2^{7} \cdot 13$ & & 101 & 2176 & $2^{7} \cdot 17$ \\
\hline \multirow[t]{2}{*}{137} & 5 & 128 & $2^{7}$ & & 109 & 6400 & $2^{8} \cdot 5^{2}$ \\
\hline & 53 & 1664 & $2^{7} \cdot 13$ & & & & \\
\hline
\end{tabular}

Note: For tables 1 and $2, p<500$ when $l=17$ or 41 and $p<$ 110 otherwise.

Table 3

$$
(d=-m \varepsilon \sqrt{l}, m=1 \text { or } 2)
$$

\begin{tabular}{r|r|r|r}
\hline$l$ & $m$ & $h^{*}$ & $f$ \\
\hline 257 & 1 & 32 & $2^{5}$ \\
337 & 1 & 256 & $2^{8}$ \\
89 & 2 & 64 & $2^{6}$ \\
113 & 2 & 32 & $2^{5}$ \\
233 & 2 & 128 & $2^{7}$ \\
\hline
\end{tabular}




\begin{tabular}{|c|c|c|c|c|c|c|c|}
\hline \multicolumn{4}{|c|}{ Table 4} & \multicolumn{4}{|c|}{ Table 4 (con't) } \\
\hline \multicolumn{4}{|c|}{$(d=-2 \varepsilon \sqrt{l} p)$} & \multicolumn{4}{|c|}{$(d=-2 \varepsilon \sqrt{l} p)$} \\
\hline$l$ & $p$ & $h^{*}$ & $f$ & $l$ & $p$ & $h^{*}$ & $f$ \\
\hline \multirow[t]{4}{*}{17} & 5 & 32 & $2^{5}$ & 113 & 7 & 320 & $2^{6} \cdot 5$ \\
\hline & 37 & 320 & $2^{6} \cdot 5$ & & 23 & 640 & $2^{7} \cdot 5$ \\
\hline & 47 & 320 & $2^{6} \cdot 5$ & & 31 & 1152 & $2^{7} \cdot 3^{2}$ \\
\hline & 61 & 256 & $2^{8}$ & & 41 & 2368 & $2^{6} \cdot 3^{7}$ \\
\hline \multirow[t]{8}{*}{41} & 3 & 32 & $2^{5}$ & & 53 & 1600 & $2^{6} \cdot 5^{2}$ \\
\hline & 11 & 256 & $2^{8}$ & & 71 & 1664 & $2^{7} \cdot 13$ \\
\hline & 13 & 128 & $2^{7}$ & & 73 & 3712 & $2^{7} \cdot 29$ \\
\hline & 19 & 512 & $2^{9}$ & 137 & 13 & 512 & $2^{9}$ \\
\hline & 23 & 256 & $2^{8}$ & & 43 & 2624 & $2^{6} \cdot 41$ \\
\hline & 31 & 640 & $2^{7} \cdot 5$ & & 67 & 3904 & $2^{6} \cdot 61$ \\
\hline & 53 & 576 & $2^{6} \cdot 3^{2}$ & & 73 & 3904 & $2^{6} \cdot 61$ \\
\hline & 67 & 512 & $2^{9}$ & 193 & 5 & 320 & $2^{6} \cdot 5$ \\
\hline \multirow[t]{4}{*}{73} & 17 & 832 & $2^{6} \cdot 13$ & & 7 & 1152 & $2^{7} \cdot 3^{2}$ \\
\hline & 37 & 576 & $2^{6} \cdot 3^{2}$ & & 13 & 3328 & $2^{8} \cdot 13$ \\
\hline & 41 & 3200 & $2^{7} \cdot 5^{2}$ & & 37 & 3392 & $2^{7} \cdot 53$ \\
\hline & 71 & 4352 & $2^{8} \cdot 17$ & & 53 & 1664 & $2^{7} \cdot 13$ \\
\hline \multirow[t]{4}{*}{89} & 11 & 512 & $2^{9}$ & & 61 & 11072 & $2^{6} \cdot 173$ \\
\hline & 17 & 320 & $2^{6} \cdot 5$ & 233 & 19 & 1280 & $2^{8} \cdot 5$ \\
\hline & 67 & 1600 & $2^{6} \cdot 5^{2}$ & & 23 & 3328 & $2^{8} \cdot 13$ \\
\hline & 73 & 1600 & $2^{6} \cdot 5^{2}$ & & 37 & 3712 & $2^{7} \cdot 29$ \\
\hline \multirow[t]{3}{*}{97} & 5 & 320 & $2^{6} \cdot 5$ & & 71 & 5248 & $2^{7} \cdot 41$ \\
\hline & 13 & 320 & $2^{6} \cdot 5$ & & 73 & 3328 & $2^{8} \cdot 13$ \\
\hline & 47 & 3200 & $2^{7} \cdot 5^{2}$ & & & & \\
\hline
\end{tabular}

\begin{tabular}{|c|c|c|c|c|c|}
\hline \multicolumn{3}{|c|}{ Table 5} & \multicolumn{3}{|c|}{ Table 5 (con't) } \\
\hline \multicolumn{3}{|c|}{$(d=-\varepsilon \sqrt{2} p)$} & \multicolumn{3}{|c|}{$(d=-\varepsilon \sqrt{2} p)$} \\
\hline$p$ & $h^{*}$ & $f$ & $p$ & $h^{*}$ & $f$ \\
\hline 47 & 32 & $2^{5}$ & 239 & 320 & $2^{6} \cdot 5$ \\
\hline 127 & 160 & $2^{5} \cdot 5$ & 257 & 160 & $2^{5} \cdot 5$ \\
\hline 223 & 160 & $2^{5} \cdot 5$ & 271 & 160 & $2^{5} \cdot 5$ \\
\hline
\end{tabular}




\begin{tabular}{|c|c|c|c|c|c|}
\hline \multicolumn{3}{|c|}{ Table 5 (con't) } & \multicolumn{3}{|c|}{ Table 5 (con't) } \\
\hline \multicolumn{3}{|c|}{$(d=-\varepsilon \sqrt{2} p)$} & \multicolumn{3}{|c|}{$(d=-\varepsilon \sqrt{2} p)$} \\
\hline$p$ & $h^{*}$ & $f$ & $p$ & $h^{*}$ & $f$ \\
\hline 367 & 160 & $2^{5} \cdot 5$ & 1279 & 640 & $2^{7} \cdot 5$ \\
\hline 431 & 320 & $2^{6} \cdot 5$ & 1423 & 1088 & $2^{6} \cdot 17$ \\
\hline 463 & 640 & $2^{7} \cdot 5$ & 1439 & 1600 & $2^{6} \cdot 5^{2}$ \\
\hline 479 & 160 & $2^{5} \cdot 5$ & 1553 & 800 & $2^{5} \cdot 5^{2}$ \\
\hline 577 & 416 & $2^{5} \cdot 13$ & 1601 & 640 & $2^{7} \cdot 5$ \\
\hline 751 & 576 & $2^{6} \cdot 3^{2}$ & 1663 & 1088 & $2^{6} \cdot 17$ \\
\hline 1039 & 800 & $2^{5} \cdot 5^{2}$ & 1759 & 1664 & $2^{7} \cdot 13$ \\
\hline 1151 & 640 & $2^{7} \cdot 5$ & 1823 & 1184 & $2^{5} \cdot 5 \cdot 17$ \\
\hline 1153 & 544 & $2^{5} \cdot 17$ & 1889 & 1184 & $2^{5} \cdot 37$ \\
\hline 1201 & 1088 & $2^{6} \cdot 17$ & 1951 & 1312 & $2^{5} \cdot 41$ \\
\hline 1217 & 512 & $2^{9}$ & & & \\
\hline
\end{tabular}

\section{REFERENCES}

1. Ezra Brown and Charles J. Parry, The 2-class group of certain biquadratic number fields, J. reine und angew, Math., 295 (1977), 61-71.

2. Gordon Pall, Discriminantal divisors of binary quadratic forms, J. Number Theory, 1 (1969), 525-533.

3. Charles J. Parry, Real quadratic fields with class number divisible by 5, Math. of Comp., 31 (1977), 1019-1029.

Received February 23, 1977.

Virginia Polytechnic Institute and State University

BLACKSBURG, VA 24061 


\section{PACIFIC JOURNAL OF MATHEMATICS}

\section{EDITORS}

RICHARD ARENS (Managing Editor)

University of California

Los Angeles, California 90024

C. W. CurTis

University of Oregon

Eugene, OR 97403

C. C. MOORE

University of California

Berkeley, CA 94720
J. DUGUNDJI

Department of Mathematics University of Southern California Los Angeles, California 90007

R. FinN AND J. Milgram Stanford University Stanford, California 94305

\section{ASSOCIATE EDITORS}

E. F. BECKENBACH

B. H. NeumanN

F. WOLF

K. YoSHIDA

\section{SUPPORTING INSTITUTIONS}

UNIVERSITY OF BRITISH COLUMBIA CALIFORNIA INSTITUTE OF TECHNOLOGY UNIVERSITY OF CALIFORNIA MONTANA STATE UNIVERSITY UNIVERSITY OF NEVADA, RENO NEW MEXICO STATE UNIVERSITY OREGON STATE UNIVERSITY UNIVERSITY OF OREGON
UNIVERSITY OF SOUTHERN CALIFORNIA

STANFORD UNIVERSITY

UNIVERSITY OF HAWAII

UNIVERSITY OF TOKYO

UNIVERSITY OF UTAH

WASHINGTON STATE UNIVERSITY

UNIVERSITY OF WASHINGTON 


\section{Pacific Journal of Mathematics \\ Vol. 78, No. $1 \quad$ March, 1978}

Simeon M. Berman, A class of isotropic distributions in $\mathbf{R}^{n}$ and their

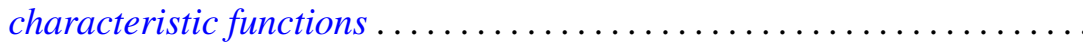

Ezra Brown and Charles John Parry, The 2-class group of biquadratic fields.

II ........................................ 11

Thomas E. Cecil and Patrick J. Ryan, Focal sets of submanifolds ....... 27

Joseph A. Cima and James Warren Roberts, Denting points in $B^{p} \ldots \ldots \ldots 41$

Thomas W. Cusick, Integer multiples of periodic continued fractions . . . . . 47

Robert D. Davis, The factors of the ramification sequence of a class of

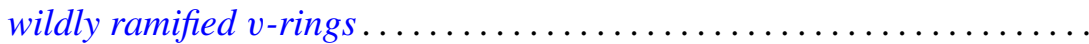

Robert Martin Ephraim, Multiplicative linear functionals of Stein

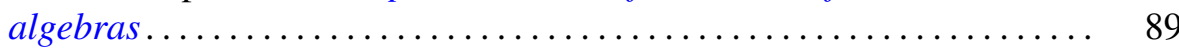

Philip Joel Feinsilver, Operator calculus . .................... 95

David Andrew Gay and William Yslas Vélez, On the degree of the splitting field of an irreducible binomial ..........................

Robert William Gilmer, Jr. and William James Heinzer, On the divisors of

monic polynomials over a commutative ring ..................

Robert E. Hartwig, Schur's theorem and the Drazin inverse .............

Hugh M. Hilden, Embeddings and branched covering spaces for three and four dimensional manifolds ............................

Carlos Moreno, The Petersson inner product and the residue of an Euler product. ...

Christopher Lloyd Morgan, On relations for representations of finite groups....

Ira J. Papick, Finite type extensions and coherence

$\mathrm{R}$. Michael Range, The Carathéodory metric and holomorphic maps on a class of weakly pseudoconvex domains ................

Donald Michael Redmond, Mean value theorems for a class of Dirichlet series

Daniel Reich, Partitioning integers using a finitely generated semigroup ...

Georg Johann Rieger, Remark on a paper of Stux concerning squarefree

numbers in non-linear sequences

Gerhard Rosenberger, Alternierende Produkte in freien Gruppen ..

Ryōtarō Satō, Contraction semigroups in Lebesgue space

Tord Sjödin, Capacities of compact sets in linear subspaces of $\mathbf{R}^{n}$

Robert Jeffrey Zimmer, Uniform subgroups and ergodic actions of exponential Lie groups......................... 